'Laboratorio de Farmacocinética y Biodisponibilidad de Medicamentos, Centro

FT Programa de Farmaco-

logía lar y Clínica, ICBM, Facultad de Medicina Universidad de Chile. 2Departamento de Pediatría División Norte Facultad de

Medicina, Universidad de

Chile.

Programa de Farmacología Molecular y Clínica, ICBM Facultad de Medicina

Universidad de Chile.

${ }^{a}$ Químico-Farmacéutico. bioquímico. Biólogo.

dAlumno de Medicina, Facul-

tad de Medicina, Universidad de Chile.

Conflicto de Intereses: $\mathrm{E}$ patrocinador del presente estudio, Corporación Farmacéutica Recalcine proporcionó el soporte financiero mediante un convenio con la Facultad de

Medicina de la Universidad de Chile. El patrocinador no tuvo participación en

la conducción, análisis y publicación de este estudio cumpliendo así las cláusulas de confidencialidad de protocolo y las normativas nacionales e internacionales, sobre estos estudios, que as

lo establecen. Los autores declaran no tener otros conflictos de intereses.

Recibido el 14 de diciembre de 2010, aceptado el 3 de mayo de 2011

Correspondencia a: Iván Saavedra S Laboratorio de Farmacociética y Biodisponibilidad de Medicamentos. Programa de Farmacología Molecular y Clínica, ICBM, Facultad de Medicina, Universidad de Chile. PO Box 70111 F: $56-2-6817756$

Fax: 56-2-6822406 E-mail: isaavedr@med. uchile.cl

\section{Estudio de biodisponibilidad relativa entre dos formulaciones orales de micofenolato mofetilo en voluntarios sanos}

\author{
IVÁN SAAVEDRA S. ${ }^{1, a}$, JAIME SASSO A. ${ }^{1, a}$, LUIS QUIÑONES S. ${ }^{1, b}$,

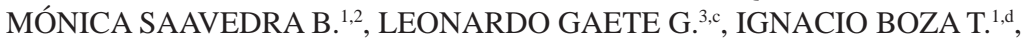 \\ CRISTÓBAL CARVAJAL H. ${ }^{1, \mathrm{~d}}$, JORGE SOTO L. ${ }^{1, \mathrm{a}, \mathrm{b}}$
}

\section{Relative bioavailability study of two oral formulations of mycophenolate mofetil in healthy volunteers}

Background: The bioequivalence of different formulations of a same pharmaceutical product must be tested empirically. Aim: To evaluate the relative bioavailability for an oral formulation of mycophenolate mofetil (MMF) (Linfonex $\left.{ }^{T M}\right)$ compared to the reference formulation (Cellcept ${ }^{T M}$ ) to determine the bioequivalence between both formulations. Material and Methods: A randomized, crossover, double-blind trial in 22 healthy male volunteers, who received a single oral dose of $1000 \mathrm{mg}$ of Linfonex and Cellcept with a washout period of 10 days. Plasma levels of the drug were determined by high performance liquid chromatography. Plasma concentrations were plotted and maximum concentration, area under the plasma concentration versus time between 0 and 12 hours after administration and area under plasma concentration curve versus time after administration between 0 and infinity, were calculated for both products. Results: The active compound, mycophenolic acid, was similarly absorbed in both formulations. No statistically significant differences were found in calculated pharmacokinetic parameters between both formulations. Conclusions: Linfonex ${ }^{T M} 500 \mathrm{mg}$ is bioequivalent to Cellcept ${ }^{T M} 500 \mathrm{mg}$.

(Rev Med Chile 2011; 139: 902-908).

Key words: Biological availability; Mycophenolate mofetil; Therapeutic equivalency.

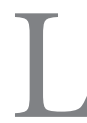
a biodisponibilidad (BD) de un fármaco es una medida de la cantidad y de la velocidad con que éste alcanza la circulación sistémica $^{1,2}$. La bioequivalencia (BE) es una medida comparativa de la biodisponibilidad de una formulación farmacéutica, entre un producto similar y el producto innovador o referente del mercado ${ }^{1,2}$, en términos de la velocidad y cantidad de principio activo que entrega. Conociendo la calidad, seguridad y eficacia de la formulación de un producto farmacéutico, el médico puede dis- poner de alternativas farmacéuticas a precios más convenientes para el paciente sin comprometer la eficacia clínica y terapéutica ${ }^{1,2}$.

La prescripción de un medicamento es el acto profesional realizado por un médico a través de la receta médica, en el entendido que su formación profesional le permite establecer cuál es la terapia adecuada para un determinado paciente. Por otra parte, la intercambiabilidad es el acto realizado por un médico de utilizar indistintamente una formulación u otra, disponiendo de elementos de 
Biodisponibilidad de dos formulaciones de micofenolato mofetilo - I. Saavedra et al

juicio suficientes para asegurar que la calidad de uno de los productos farmacéuticos es comparable o similar a la del otro².

El ácido micofenólico (MPA) es un fármaco que se administra como la pro-droga micofenolato mofetilo (MMF) y es ampliamente usado en el trasplante de órganos como un inmunosupresor para prevenir el rechazo agudo y de órganos trasplantados. MPA es un compuesto obtenido a partir de la fermentación de diversas especies Penicillum $y$ un potente inhibidor reversible no competitivo de la inosina monofosfato deshidrogenasa eucariótica. MPA interfiere en la vía síntesis de novo de guanosina y subsecuentemente con la replicación del ADN. La actividad del MPA es altamente específica por linfocitos porque su proliferación depende enteramente de la síntesis de novo para purinas ${ }^{3}$.

La farmacocinética del MPA ha sido extensamente estudiada en voluntarios sanos y en pacientes trasplantados. Un análisis exhaustivo de la literatura refleja una amplia variabilidad en los parámetros farmacocinéticos del fármaco, atendiendo a las dosis utilizadas, tiempos de muestreo y condición clínica de los pacientes ${ }^{4-8}$. MMF es absorbido de forma rápida y es ampliamente metabolizado presistémicamente a MPA ${ }^{8}$. La BD del MMF es, en promedio, de un $94 \%$ y la eliminación se realiza fundamentalmente a través de la orina como un derivado glucurónido del fármaco ${ }^{8}$. Los alimentos no alteran la magnitud de la absorción, reflejada como área bajo la curva. Sin embargo, la presencia de alimentos reduce la concentración máxima $\left(\mathrm{C}_{\text {máx }}\right)$ hasta $40 \%{ }^{8}$.

Existe una gran cantidad de factores que pueden afectar la $\mathrm{BD}$ de un fármaco, entre los cuales mencionamos las características físico-químicas del principio activo, la calidad y tipo (cápsula, comprimidos, grageas, etcétera) de la forma farmacéutica, la calidad y tipo de los excipientes, el proceso de fabricación, el tipo y calidad de empaque, la estabilidad del producto, etcétera. Por lo tanto, demostrar que una formulación es intercambiable por otra, involucra el desarrollo de un estudio de $\mathrm{BD} / \mathrm{BE}^{9}$ y para que esta propiedad sea replicable en todos los lotes de producción, la industria debe estar validada por la autoridad sanitaria en las Buenas Prácticas de Manufactura (GMP, sigla en inglés) y en el proceso de fabricación específico del medicamento en estudio.

Para garantizar el acceso y disponibilidad a medicamentos eficaces y seguros a la población, la política nacional de medicamentos en la Reforma de Salud dicta que los medicamentos comercializados en Chile deben cumplir estándares de eficacia y seguridad y demostrar equivalencia terapéutica con un producto de referencia oficial o comparador que es definido y certificado por la autoridad sanitaria ${ }^{10}$. Al ser el MMF una droga con metabolismo de primer paso elevado $(>70 \%)^{11}$, esta equivalencia debe demostrarse a través de estudios in vivo ${ }^{10}$.

El objetivo del presente trabajo fue evaluar, mediante un estudio doble ciego y cruzado, en 22 voluntarios sanos, la biodisponibilidad relativa de una formulación oral de micofenolato mofetilo, comprimidos de $500 \mathrm{mg}$, con respecto al producto de referencia, después de una sola dosis de 1.000 $\mathrm{mg}$. La formulación de referencia fue el producto innovador Cellcept ${ }^{\circledast}$ y el producto test fue el producto Linfonex ${ }^{\oplus}$.

\section{Voluntarios, Materiales y Métodos}

El protocolo del estudio fue aprobado por el Comité de Ética de la Investigación en Seres $\mathrm{Hu}-$ manos de la Facultad de Medicina de la Universidad de Chile, con fecha 26 de mayo de 2005. El presente estudio fue realizado en el Laboratorio de Farmacocinética de la Facultad de Medicina de la Universidad de Chile. En todo momento, se siguieron las recomendaciones para guiar los estudios en seres humanos emanados de la declaración de Helsinki ${ }^{12}$ y las Buenas Prácticas Clínicas ${ }^{13}$.

\section{Voluntarios}

Luego de haber sido informados por el médico responsable acerca de los propósitos, riesgos y posibles beneficios del estudio, todos los voluntarios firmaron el consentimiento informado. Los voluntarios fueron seleccionados luego de ser sometidos a una entrevista medica, un completo examen físico y a un conjunto de pruebas de laboratorio (drogas de abuso, hemograma y VHS, orina completa, VIH, hepatitis, VDRL, creatinemia, glicemia, uremia, proteinemia, fosfatasas alcalinas, bilirubinemia, transaminasas oxaloacética y pirúvica).

En el estudio participaron 22 voluntarios varones sanos, cuyas características antropométricas se resumen a continuación: peso (promedio \pm desviación estándar) $74,70 \pm 8,84 \mathrm{~kg}$; altura 
$175,73 \pm 8,49 \mathrm{~cm}(\mathrm{IMC}=24,29 \pm 3,36)$ y $28,41 \pm$ 8,99 años de edad.

Durante el estudio los voluntarios estuvieron permanentemente en contacto con el médico del centro de investigación y en los días de tratamiento fueron atendidos por el equipo profesional del Laboratorio. Su alimentación, a saber desayuno, almuerzo, meriendas y cena, fue diseñada por un profesional Nutricionista sobre la base de lo aconsejado por el médico.

\section{Productos farmacéuticos}

Las formulaciones de micofenolato mofetilo estudiadas correspondieron a: Linfonex ${ }^{\infty}$ (A), proporcionado y fabricado por Laboratorios Recalcine S.A., cuya planta de producción cuenta con certificación GMP, comprimidos recubiertos de $500 \mathrm{mg}$, número de serie F04375, el cual fue considerado como producto test y Cellcept ${ }^{\circ}$ (B), comprado en el mercado norteamericano, fabricado por Hoffmann La Roche, USA, tabletas de $500 \mathrm{mg}$, número de lote U4354, el cual fue considerado como producto referencia. El proceso de producción del producto test se encuentra validado, cabe destacar que esta condición es indispensable para concluir la bioequivalencia entre ambos productos.

\section{Metodología}

Los productos farmacéuticos se administraron mediante un estudio aleatorio, cruzado, comparativo y doble ciego.

Se administró una dosis oral única de 1.000 $\mathrm{mg}$, en ayunas, con $250 \mathrm{~mL}$ de agua, estando el voluntario de pie. No se permitió la ingesta de alimentos hasta después de $2 \mathrm{~h}$ de la administración. Durante el transcurso del estudio, los voluntarios recibieron dietas estandarizadas y se les prohibió el consumo de café, alcohol o tabaco. Durante el primer período de estudio, once voluntarios recibieron el producto test $y$ los otros once el producto referencia. En el segundo período de estudio, los voluntarios se cruzaron, cambiando el producto farmacéutico. Ambos períodos estuvieron separados por 10 días (transcurridas más de 5 tiempos de vida media del fármaco).

Dos Enfermeros Universitarios colocaron a cada voluntario una bránula antebraquial (Beckton \& Dickinson, $18 \mathrm{G}$ ) provista de una llave de tres pasos, la que mantuvieron en forma permeable con heparina sódica diluida como anticoagulante para la extracción de la sangre.

Las muestras sanguíneas de $5 \mathrm{~mL}$, se colectaron a tiempo 0 (antes de administrar la droga) y a 0,$5 ; 1 ; 1,5 ; 2 ; 2,5 ; 3 ; 4 ; 6 ; 8 ; 10$ y 12 h post dosis. Las muestras fueron centrifugadas a $2.000 \mathrm{rpm}$ durante $10 \mathrm{~min}$ a $4^{\circ} \mathrm{C}$ y el plasma se almacenó a $-20^{\circ} \mathrm{C}$ hasta el momento de su análisis.

\section{Método analítico}

Las determinaciones de las concentraciones plasmáticas de MPA se realizaron mediante cromatografía líquida de alta resolución (HPLC) con detección UV, este método fue validado de acuerdo a la guía de la FDA para verificar y documentar su validez analítica ${ }^{14}$. Se utilizó como referencia el método de Svensson ${ }^{15}$ el cual permite la detección y cuantificación del ácido micofenólico. Para extraer la droga desde la muestra se utilizó el siguiente Procedimiento: a $100 \mu \mathrm{L}$ de plasma se adicionó $200 \mu \mathrm{L}$ de ácido fosfórico $0,1 \mathrm{M}$ disueltos en acetonitrilo, luego la mezcla se agitó en vortex durante un minuto y se centrifugó por 5 minutos a 5.000 $\mathrm{rpm}$, finalmente se inyectaron al cromatógrafo 20 $\mu \mathrm{L}$ del sobrenadante.

El análisis cromatográfico se realizó en un equipo HPLC marca Elite LaChrom, Merck-Hitachi. La columna analítica fue del tipo C18-LichroCart $125 \times 4 \mathrm{~mm}$. La fase móvil consistió en una mezcla de ácido fosfórico 0,04 M, pH 2,1 y acetonitrilo (70:30), se utilizó un flujo de $1,5 \mathrm{~mL} / \mathrm{min}$ y una longitud de onda de $215 \mathrm{~nm}$. El MPA tuvo un tiempo de retención de 10,3 minutos.

La linealidad de la metodología fue validada en un rango de concentración de 0,5 a $60 \mu \mathrm{g} / \mathrm{mL}$ de MPA en plasma humano, obteniéndose un coeficiente de correlación de 0,995. El límite de detección se estableció en $0,3 \mu \mathrm{g} / \mathrm{mL}$. La precisión y la exactitud de esta metodología fue evaluada realizando análisis en replicado $(n=5)$ de los controles de calidad (QC) de 4,0; 10 y $40 \mu \mathrm{g} / \mathrm{mL}$. El error relativo (ER) de la metodología fue de 1,0;0,2 y $1,3 \%$, respectivamente. El coeficiente de variación (CV) fue de 2,7; 0,81 y 0,50\%, respectivamente. Tanto el ER para evaluar exactitud y el CV para evaluar precisión fueron $<15 \%$.

Los reactivos y solventes fueron adquiridos en Merck S.A.; el estándar de ácido micofenólico fue adquirido en la USP y fue proporcionado por Laboratorio Recalcine, con él se preparó una solución estándar de $1 \mathrm{mg} / \mathrm{mL}$ en metanol. 


\section{Análisis farmacocinético y estadístico}

Se empleó un método farmacocinético no compartimental para determinar los parámetros del MPA. Los parámetros denominados primarios, utilizados para establecer bioequivalencia fueron: concentración máxima $\left(\mathrm{C}_{\text {máx }}\right)$ y área bajo la curva de concentraciones plasmáticas hasta 12 horas $\left(\mathrm{ABC}_{0-12}\right)$ y hasta tiempo infinito $\left(\mathrm{ABC}_{0-\infty}\right)$. Los parámetros secundarios fueron tiempo máximo $\left(t_{\text {máx }}\right)$, constante de eliminación $\left(k_{e}\right) . C_{\text {máx }} \mathrm{y}_{\text {máx }}$ fueron obtenidos directamente desde la curva de concentración plasmática de MPA versus tiempo. $\mathrm{ABC}_{0-12}$ fue calculado usando la regla de los trapezoides, $\mathrm{ABC}_{0-\infty}$ fue calculado usando la siguiente fórmula: $\mathrm{ABC}_{0-\infty}=\mathrm{ABC}_{0-12}+C_{12} / \mathrm{k}_{\mathrm{e}}$, donde $C_{12}$ representa la última concentración medida. $\mathrm{La}_{\mathrm{e}}$ corresponde a la pendiente obtenida de la regresión de mínimos cuadrados de la porción lineal de la curva concentración plasmática logaritmo natural transformada versus tiempo. Todos los parámetros farmacocinéticos fueron calculados utilizando la aplicación "pkexamine" del paquete estadístico STATA 10,0.

Para determinar la normalidad de la distribución de los parámetros farmacocinéticos se aplicó la prueba de Shapiro-Wilk ${ }^{16}$. Para establecer las posibles diferencias entre los parámetros farmacocinéticos primarios $\left(\mathrm{C}_{\text {máx }}\right.$ y $\left.A B C\right)$, se utilizó el test de análisis de varianza multifactorial (ANOVA), estimándose una diferencia estadísticamente significativa para valores de $\mathrm{p} \leq 0,05$.
Siguiendo las recomendaciones de la FDA, se determinaron los intervalos de confianza de 90\% para la razón entre los parámetros farmacocinéticos promedio obtenidos con ambas formulaciones, luego de transformar estos valores a logaritmo natural ${ }^{17}$. Para el cálculo de la BE se utilizaron valores límites entre 80 a 125\% con relación al producto de referencia ${ }^{17}$. Además para establecer la bioequivalencia se realizó el análisis "two one-side test" (TOST) de Schuirman ${ }^{18}$. Todos estos análisis se realizaron utilizando la aplicación "pkequiv" de STATA 10.0.

\section{Reacciones adversas}

Durante ambos períodos de estudio todos los voluntarios estuvieron en observación médica permanente de manera tal de atender cualquier evento adverso, con o sin relación a la administración del medicamento.

\section{Resultados}

No se observaron reacciones adversas al fármaco en los voluntarios con ninguno de los dos medicamentos. La encuesta realizada por el médico iba dirigida a detectar efectos adversos tales como: cefaleas, dispepsia, diarreas y somnolencia.

En la Figura 1 se presentan las curvas de concentración plasmática promedio versus tiempo obtenido con cada formulación en los 22 voluntarios.

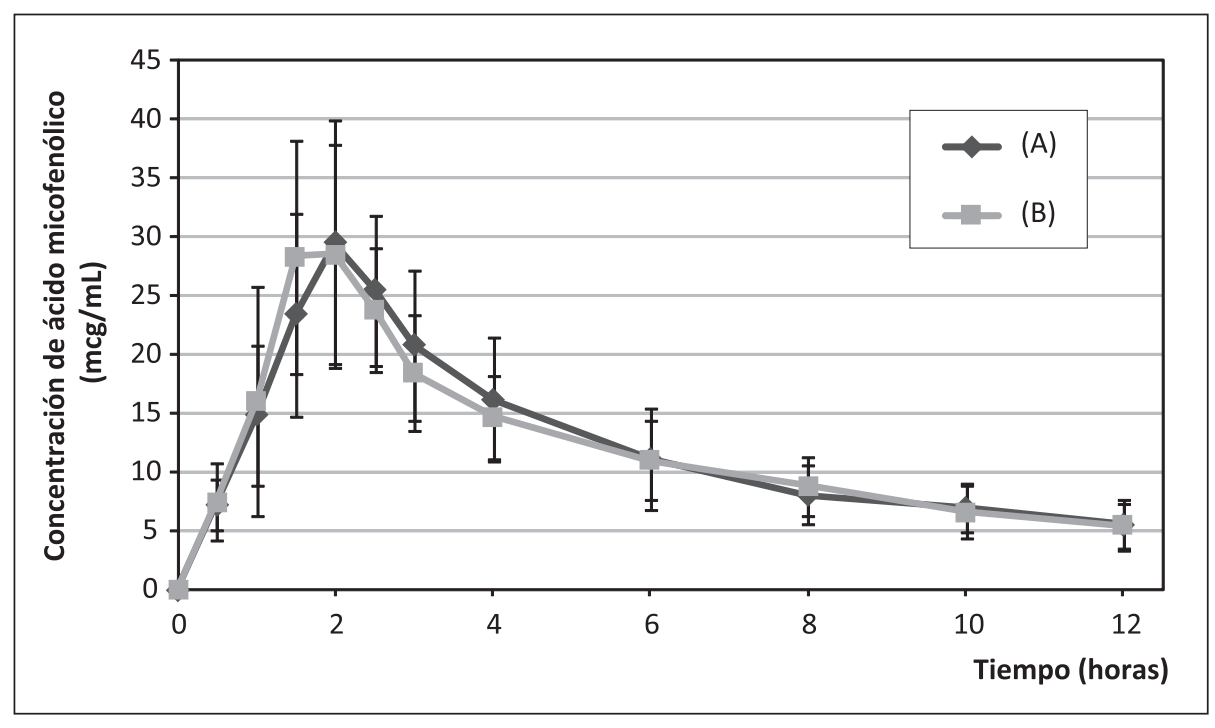

Figura 1. Niveles plasmáticos promedio de ácido micofenólico en voluntarios sanos $(n=22)$. *Cada punto representa el promedio de los 22 voluntarios y su desviación estándar. $A=$ Linfonex $^{\circledR} y$ $\mathrm{B}=$ Cellcept $^{\circledR}$. 
Biodisponibilidad de dos formulaciones de micofenolato mofetilo - I. Saavedra et al

Tabla 1. Parámetros farmacocinéticos promedio de ácido micofenólico en 22 voluntarios, luego de recibir una dosis de $1.000 \mathrm{mg}$

\begin{tabular}{|lcc|}
\hline & Linfonex ${ }^{\circledR}$ (promedio \pm D.E.) & Cellcept ${ }^{\circledR}$ (promedio \pm D.E.) \\
\hline$C_{\text {máx }}(\mu \mathrm{g} / \mathrm{mL})$ & $30,93 \pm 8,46$ & $33,1 \pm 9,56$ \\
$T_{\text {máx }}(\mathrm{h})$ & $2,11 \pm 0,26$ & $1,70 \pm 0,37$ \\
$A B C_{0-12}\left(\mu \mathrm{g}^{*} \mathrm{~h} / \mathrm{mL}\right)$ & $147,36 \pm 32,27$ & $145,90 \pm 21,67$ \\
$A B C_{0-1 \mathrm{NF}}\left(\mu \mathrm{g}^{*} \mathrm{~h} / \mathrm{mL}\right)$ & $226,77 \pm 109,57$ & $217,22 \pm 75,35$ \\
\hline
\end{tabular}

$\mathrm{C}_{\text {máx }}=$ concentración máxima, $\mathrm{t}_{\text {máx }}=$ tiempo máximo, $\mathrm{ABC}_{0-12}=$ área bajo la curva de concentraciones plasmáticas hasta 12 horas y $\mathrm{ABC}_{0-\infty}=$ área bajo la curva de concentraciones plasmáticas hasta tiempo infinito.

Tabla 2. Análisis de varianza (ANOVA) para un estudio cruzado $2 \times 2$

\begin{tabular}{|lccc|}
\hline $\begin{array}{l}\text { Fuente de } \\
\text { variación }\end{array}$ & $\begin{array}{c}\text { LnCmáx } \\
\text { (p-value) }\end{array}$ & $\begin{array}{c}\text { LnABC } \\
\mathbf{0 - 1 2} \\
\text { (p-value) }\end{array}$ & $\begin{array}{c}\text { LnABC } \\
\text { 0-inf } \\
\text { (p-value) }\end{array}$ \\
\hline $\begin{array}{l}\text { Interindividual } \\
\text { - Efecto Secuencia }\end{array}$ & 0,6291 & 0,2434 & 0,2277 \\
\hline $\begin{array}{l}\text { Intraindividual } \\
\text { - Efecto Tratamiento } \\
\text { - Efecto Período }\end{array}$ & 0,3238 & 0,9145 & 0,9923 \\
\hline
\end{tabular}

Los parámetros farmacocinéticos $\mathrm{ABC}_{0-12}$, $\mathrm{ABC}_{0-\infty}, \mathrm{C}_{\text {máx }} \mathrm{y} \mathrm{t}_{\text {máx }}$ de ácido micofenólico para ambas formulaciones, se muestran en la Tabla 1. Con el propósito de evaluar diferencias estadísticamente significativas en los parámetros farmacocinéticos concentración dependiente $\left(\mathrm{C}_{\text {máx }} \mathrm{y}\right.$ $\mathrm{ABC}$ ) y logaritmo transformados se incluyeron los factores de variabilidad período, secuencia y tratamiento en el análisis de varianza (ANOVA); estos resultados se muestran en la Tabla 2, los parámetros no presentaron diferencias $(p>0,05)$.

$\mathrm{Al}$ aplicar las pruebas estadísticas sugeridas por la FDA, se encontró que las formulaciones bajo estudio son bioequivalentes (Tabla 3 ) y por lo tanto, pueden considerarse como equivalentes terapéuticos. En otras palabras, las diferencias caen dentro del límite 80-125\% y los resultados obtenidos para el test de Schuirman $(\mathrm{p}<0,05)$ permiten rechazar la hipótesis nula de bio-inequivalencia.

\section{Discusión}

La bioequivalencia entre dos formulaciones farmacéuticas, alude al concepto de la intercambiabilidad entre ellas. Los productos genéricos son, de acuerdo a la Organización Mundial de la Salud, "productos farmacéuticos de múltiples orígenes intercambiables"19,20. Para fines de intercambiabilidad de una formulación por otra, es necesario conocer las características de solubilidad y permeabilidad de la droga; para cierto tipo de fármacos (muy solubles y muy permeables), se considera que la aplicación del ensayo de perfil de disolución comparativa arroja suficiente evidencia para documentar la bioequivalencia entre dos formulaciones; en el otro extremo, para fármacos poco solubles y poco permeables, un estudio de BE in vivo, en voluntarios sanos, constituye la forma de documentar la intercambiabilidad ${ }^{9}$.

En general, la evaluación de la BE se basa en la medición de las concentraciones de fármaco sin

Tabla 3. Pruebas de bioequivalencia

\begin{tabular}{|lllll|}
\hline & \multicolumn{2}{c}{ Límites del Test (IC90\%) } & \multicolumn{2}{c|}{ Two One-Side Test (TOST) } \\
\hline & Límite inferior & Límite superior & Límite superior & Límite superior \\
\hline Ln Cmáx & $94,71 \%$ & $101,38 \%$ & $<0,0001$ & $<0,0001$ \\
\hline $\operatorname{Ln} A B C_{0 a 12}$ & $98,15 \%$ & $101,63 \%$ & $<0,0001$ & $<0,0001$ \\
\hline $\operatorname{Ln} A B C_{\text {oa } \infty}$ & $96,55 \%$ & $103,41 \%$ & $<0,0001$ & $<0,0001$ \\
\hline
\end{tabular}


Biodisponibilidad de dos formulaciones de micofenolato mofetilo - I. Saavedra et al

modificar liberado desde la forma farmacéutica, más que de la determinación de la concentración de metabolitos ${ }^{10}$. En algunas situaciones particulares, podría ser más importante medir la concentración del metabolito que la del compuesto sin modificar, esto puede ocurrir en las siguientes situaciones: la medición de las concentraciones del metabolito terapéuticamente activo es aceptable si el producto estudiado es un profármaco, cuando las concentraciones del fármaco sin metabolizar son demasiado bajas para permitir una cuantificación analítica confiable en plasma por un plazo adecuado de tiempo ${ }^{10}$. MMF seguido de la administración oral es absorbido y transformado mediante metabolismo pre-sistémico a su metabolito activo, $\mathrm{MPA}^{21}$, por lo tanto, MMF es la pro-droga inactiva del MPA y además las concentraciones plasmáticas de MMF son muy pequeñas y deben ser medidas mediante una técnica muy sensible tal como la cromatografía líquida acoplada a un espectrómetro de masas.

De acuerdo a nuestros resultados y considerando aquellos parámetros farmacocinéticos que reflejan la cantidad de fármaco absorbido por el organismo y la velocidad a la cual se realiza este proceso, los productos estudiados son bioequivalentes. En relación a los resultados presentados en la Tabla 3, el intervalo de confianza al 90\% de la razón de los parámetros transformados logarítmicamente, cae dentro de los límites recomendados de $80-125 \%$, por consiguiente los productos Linfonex $^{\oplus}$ y Cellcept ${ }^{\oplus}$ pueden ser considerados como bioequivalentes. Es importante señalar que estos límites son los sugeridos por los organismos reguladores de países donde se ha desarrollado una guía para establecer bioequivalencia, tales como FDA-EE.UU., HPB-Canadá, EMEA-Europa, ANVISA-Brasil e ISP-Chile.

Cabe destacar que para obtener resultados clínicos aceptables con este medicamento, se requiere de individualización de las dosis de MMF; sin embargo, el monitoreo puede ser difícil debido a la gran variabilidad farmacocinética inter individual, básicamente debido a la variabilidad en el proceso de absorción. No obstante, el monitoreo a través de la medición de niveles plasmáticos de MPA optimiza la eficacia del tratamiento inmunosupresor.

Finalmente, este estudio consiste en una evidencia científica, aceptada internacionalmente, que permite establecer intercambiabilidad entre una formulación similar y el innovador o referente, lo que constituye una necesidad actual, particularmente en nuestro país, en donde existen una gran cantidad de medicamentos sin estos estudios. Ello permite optimizar la terapia farmacológica, tener un universo de medicamentos de calidad, seguridad y eficacia comprobada, equivalente al medicamento innovador y contribuir a mejorar la salud pública.

Agradecimientos: Los autores desean agradecer la colaboración y excelente calidad técnica del Sr. Santiago Leyton M. (Q.E.P.D) y del personal biomédico involucrado en el estudio.

\section{Referencias}

1. Guidance for Industry. Bioavailability and bioequivalence studies for orally administered drug products. General Considerations. U.S. Department of Health and Human Services. Food and Drug Administration. Center for Drug Evaluation and Research (CDER). March 2003. http://www.fda.gov/Drugs/GuidanceComplianceRegulatoryInformation/ Guidances/ucm200249.htm

2. Gaete LE, Solís GJ, Venegas FP, Carrillo CMJ, Schatloff BO, Saavedra SI. Estudio de biodisponibilidad comparativa de dos formulaciones de risperidona existentes en el mercado chileno. Rev Med Chile 2003; 131: 527-34.

3. Ransom JT. Mechanism of action of mycophenolate mofetil. Ther Drug Monit 1995; 17: 681-4.

4. Masri MA, Rizk S, Attia ML, Barbouch H, Rost M. Bioavailability of a new generic formulation of mycophenolate mofetil MMF 500 versus CellCept in healthy adult volunteers. Transplant Proc 2007; 39 (4): 1233-6.

5. Estevez-Carrizo FE, Parrillo S, Cedres M, Estevez-Parrillo FT, Rodríguez P. Comparative bioavailability of two oral formulations of mycophenolate mofetil in healthy adult Uruguayan subjects: a case of highly variable rate of drug absorption. Int J Clin Pharmacol Ther 2010; 48 (9): 621-7.

6. Almeida S, Filipe A, Neves R, Spínola AC, Tanguay M, Ortuño J, et al. Mycophenolate mofetil 500-mg tablet under fasting conditions: single-dose, randomizedsequence, open-label, four-way replicate crossover, bioequivalence study in healthy subjects. Clin Ther 2010; 32 (3): 556-74.

7. Zhang Q, Tao Y, Zhu Y, Zhu D. Bioequivalence and pharmacokinetic comparison of two mycophenolate mofetil formulations in healthy Chinese male volunteers: an open-label, randomized-sequence, single-dose, two-way crossover study. Clin Ther 2010; 32 (1): 171-8.

8. Fulton B, Markham A. Mycophenolate mofetil. A review 
Biodisponibilidad de dos formulaciones de micofenolato mofetilo - I. Saavedra et al

of its pharmacodynamic and pharmacokinetic properties and clinical efficacy in renal transplantation. Drugs 1996; 51(2): 278-98.

9. Saavedra I. Estudios de biodisponibilidad para establecer bioequivalencia de medicamentos. Cuadernos Médicos Sociales 2010; 50 (1): 11-23.

10. Guía Técnica G-BIOF 01: Estudios de Biodisponibilidad Comparativa con producto de Referencia para establecer equivalencia terapéutica. Instituto de Salud Pública de Chile (ISP), 2007.

11. Cellcept ${ }^{\oplus}$, Mycophenolate Mofetil. U.S. Food and Drug Administration (FDA)..http://www.accessdata.fda.gov/ drugsatfda_docs/label/2009/050722s021,050723s019,05 0758s019,050759s024lbl.pdf. Último acceso noviembre $11,2010$.

12. Declaration of Helsinki (1964). Recommendations guiding physicians in biomedical research involving human subjects. BMJ 1996; 313: 1448.

13. Guidance for Industry. "E6 Good Clinical Practice: consolidate guidance" US. Department of Health and Human Services, FDA, Center for Drug Evaluation and Research, April 1996. ICH.

14. Guidance for Industry. Bioanalytical method validation. Food and Drug Administration. Department of Health and Human Services. Center for Drug Evaluation and Research, May 2001.
15. Stevensson JO, Brattström C, Säwe J. A simple HPLC method for simultaneous determination of mycophenolic acid and mycophenolic acid glucuronide in plasma. Ther Drug Monit 1999; 21 (3): 322-4.

16. Shapiro SS \& Wilk MB. An analysis of variance test for normality (complete samples). Biometrika 1965; 52: 591-611.

17. Guidance for Industry. Guidance of statistical approaches to establishing bioequivalence. Food and Drug Administration. February 2001.

18. Schuirman DJ. A comparison of the two one-sided tests procedure and the power approach for assessing the equivalence of average bioavailability. J Pharmacokinet Biopharm 1987; 15: 657-80.

19. Multisource (Generic) Pharmaceuticals Products: Guidelines On Registration Requirements to Establish Interchangeability, WHO Expert Committee On Specifications for Pharmaceutical Preparations, Thirty Four Report (WHO Technical Report Series, No 937), WHO 2006.

20. Meredith PA. Generic Drugs. Therapeutic equivalence. Drug Safe 1996; 15: 233-42.

21. Lévesque E, Benoit-Biancamano MO, Delage R, Couture F, Guillemette C. Pharmacokinetics of mycophenolate mofetil and its glucuronide metabolites in healthy volunteers. Pharmacogenomics 2008; 9: 869-79. 